\title{
THE EFFECT OF TRIPLE-JUNCTION DRAG ON GRAIN GROWTH
}

\author{
G. GOTTSTEIN ${ }^{1} \uparrow$, A. H. KING ${ }^{2}$ and L. S. SHVINDLERMAN ${ }^{1,3}$ \\ ${ }^{1}$ Institut für Metallkunde und Metallphysik, RWTH Aachen, D-52056 Aachen, Germany, ${ }^{2}$ School of \\ Materials Engineering, Purdue University, West Lafayette, IN 47907-1289, USA and ${ }^{3}$ Institute of Solid \\ State Physics, Russian Academy of Science, Chernogolovka, Moscow distr. 142432, Russia
}

(Received 17 March 1999; accepted 4 October 1999)

\begin{abstract}
Current theories of grain growth presume that grain boundary migration is the rate-limiting step, and either explicitly or implicitly assume that triple junctions can always move with sufficient speed to accommodate the changing positions of the grain boundaries. Following from some recent observations of triple-junction drag effects in tricrystals of zinc and in molecular dynamics models, an analytical theory is developed to explore the effects of triple-junction drag upon grain growth, for a two-dimensional solid. The theory is developed in the framework of the Von Neumann-Mullins formulation, and demonstrates that drag effects operating exclusively at the triple junctions result in a retardation of grain growth. The stability of six-sided grains in the isotropic, drag-free case of the Von Neumann-Mullins analysis is successively extended to grains of $6 \pm N$ sides, where $N$ increases with the strength of the triple-junction drag. (C) 2000 Acta Metallurgica Inc. Published by Elsevier Science Ltd. All rights reserved.
\end{abstract}

Keywords: Triple junction; Mobility; Grain boundaries; Grain growth

\section{INTRODUCTION}

The fact that a line (or column) of intersection of three boundaries constitutes a system with specific thermodynamic properties was realized more than a hundred years ago [1]. However, the kinetic properties of this subject, in particular the mobility of triple junctions, were ignored up to now. Although the number of triple junctions in polycrystals is comparable in magnitude with the number of boundaries, all peculiarities in the behavior of polycrystals during grain growth were solely attributed to the motion of grain boundaries so far. It was tacitly assumed in theoretical approaches, computer simulations and interpretation of experimental results, that triple junctions do not disturb grain boundary motion and that their role in grain growth is reduced to preserve the thermodynamically prescribed equilibrium angles at the lines (or the points for two-dimensional systems) where boundaries meet. Inspired by experimental results on bicrystals, advanced vertex models have recently incorporated triple-junction mobility as an additional kinetic parameter and thus were successful in reproducing experimental results of triple junction and grain boundary motion in two-dimensional

$\dagger$ To whom all correspondence should be addressed. systems [2]. The most prominent example of how this assumption determines the fundamental concepts of grain structure evolution gives the Von Neumann-Mullins relation [3, 4]. No doubt this relation forms the basis for practically all theoretical and experimental investigations as well as computer simulations of microstructure evolution in twodimensional polycrystals in the course of grain growth [5-7]. This relation is based on three essential assumptions, namely: (i) all grain boundaries possess equal mobilities and surface tensions, irrespective of their misorientation and crystallographic orientation of the boundaries; (ii) the mobility of a grain boundary is independent of its velocity; (iii) the third assumption relates directly to the triple junctions, namely, they do not affect grain boundary motion; therefore, the contact angles at triple junctions are in equilibrium and, due to the first assumption, are equal to $120^{\circ}$.

\section{EFFECT OF TRIPLE JUNCTIONS}

Let us consider a two-dimensional grain with an area $S$ (Fig. 1), using the assumptions given above. The rate of change of the grain area during grain growth, where the driving force of grain boundary migration is the free energy of grain boundaries, can be written as [3] 


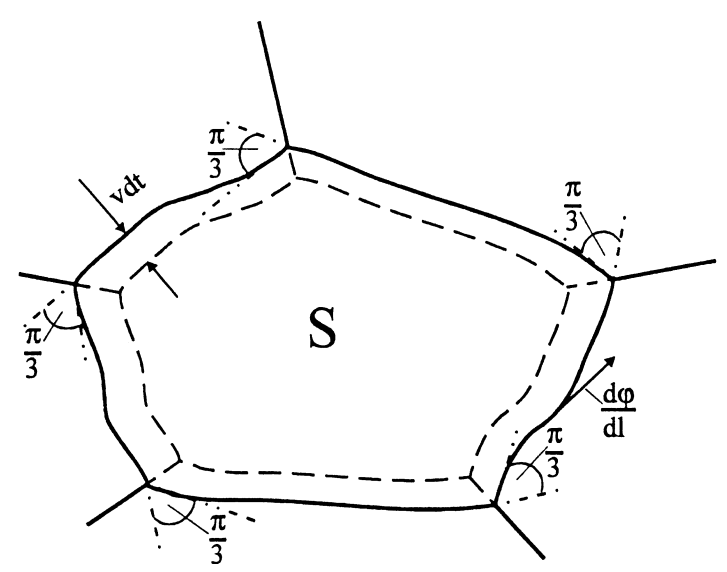

Fig. 1. Definition of parameters for a calculation of the rate of grain area change.

$$
\frac{\mathrm{d} S}{\mathrm{~d} t}=A_{\mathrm{b}} \oint \mathrm{d} \varphi
$$

If the grain were bordered by a smooth line, the integral in equation (1) would equal $2 \pi$. However, owing to the discontinuous angular change at every triple junction, the angular interval $\Delta \varphi=\pi / 3$ is subtracted from the total value $2 \pi$ for each triple junction. Consequently

$$
\frac{\mathrm{d} S}{\mathrm{~d} t}=-A_{\mathrm{b}}\left(2 \pi-\frac{n \pi}{3}\right)=\frac{A_{\mathrm{b}} \pi}{3}(n-6)
$$

where $n$ is the number of triple junctions for each respective grain, i.e. the topological class of the grain. Thus, the rate of area change is independent of the shape of the boundaries and determined by the topological class $n$ only. Grains with $n>6$ will grow and those with $n<6$ will disappear [4].

The existence of triple junctions drastically affects the kinetics of grain growth. To discuss this problem quantitatively the mobility of a triple junction should be measured. However, the steady-state motion of a grain boundary system with a triple junction when the curved boundary holds its shape during motion, and the entire system moves with the same velocity $V$, is only possible in a very narrow class of geometrical configurations. Two of these special boundary systems were investigated in Refs [8-10] under three main assumptions. Two of them comply with assumptions (1) and (2) of the Von Neumann-Mullins consideration, while the third one is determined by equation (2): the normal grain boundary displacement rate $v$ is proportional to the grain boundary curvature $K$.

As shown in Ref. [8], the model grain boundary system (Fig. 2) can move steadily and the analysis of its motion permits us to understand the influence of the finite mobility of a triple junction on the migration of grain boundaries. As it was shown in Ref. [9] the problem for a shape and velocity $V$ of moving grain boundary with a triple junction (con-

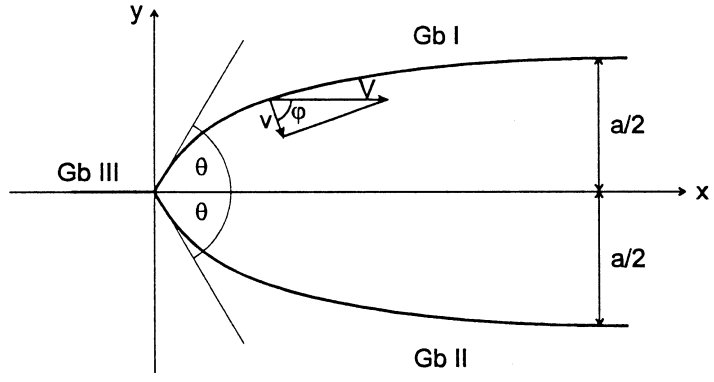

Fig. 2. Configuration of grain boundaries at a triple junction during steady-state motion for $n<6$.

figuration in Fig. 2) can be strictly solved analytically.

The solution for the shape of a moving boundary can be expressed as

$$
\begin{gathered}
y(x)=\xi \arccos \left(\mathrm{e}^{-x / \xi+c_{1}}\right)+c_{2} \\
\xi=a / 2 \Theta \\
c_{1}=\ln (\sin \Theta) \\
c_{2}=-\xi(\pi / 2-\Theta) .
\end{gathered}
$$

The velocity $V$ of steady-state motion of the system is

$$
V=\frac{2 \Theta m_{\mathrm{b}} \sigma}{a}
$$

A driving force $\sigma(2 \cos \Theta-1)$ acts on the triple junction from the curved boundaries. Introducing the mobility of the triple junction $m_{\mathrm{Tj}}$, its velocity reads

$$
V_{\mathrm{Tj}}=m_{\mathrm{Tj}} \sigma(2 \cos \Theta-1) .
$$

Due to the fact that the driving force acting on the grain boundary is a pressure and the driving force on the triple junction is a force, the dimensions of grain boundary and triple-junction mobility are different, so that their ratio $m_{\mathrm{b}} / m_{\mathrm{Tj}}$ has the dimension of a length.

The steady-state value for the angle $\Theta$ can be found from the equation

$$
\frac{2 \Theta}{2 \cos \Theta-1}=\frac{m_{\mathrm{Tj}} a}{m_{\mathrm{b}}}=\Lambda
$$

If a triple junction is mobile and does not drag grain boundary motion, the criterion $\Lambda \rightarrow \infty$ and $\Theta \rightarrow \pi / 3$, i.e. the equilibrium angular value at a triple junction in the uniform grain boundary model. In contrast, however, when the mobility of the triple junction is relatively low (strictly speaking, when $m_{\mathrm{Tj}} a \ll m_{\mathrm{b}}$ ) then $\Theta \rightarrow 0$ (Fig. 3). It should be stressed that the angle $\Theta$ is strictly defined by the dimensionsless criterion $\Lambda$, which, in turn, is a func- 


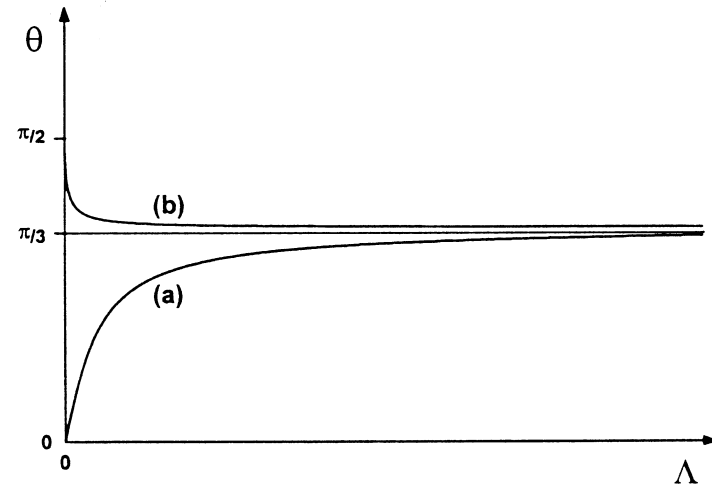

Fig. 3. Angle $\Theta$ as a function of $\Lambda$ : (a) for $n<6$, equation (6); (b) for $n>6$, equation (12).

tion of not only the ratio of triple junction and grain boundary mobility, but of the grain size as well.

Experimental investigations [9] were based on the considered grain boundary system (Fig. 2). It was shown that triple junctions do possess a finite mobility. The experimentally observed shape of a moving grain boundary fits the theoretically calculated [equation (3)] one quite well; the velocities $V$ [equations (4) and (5)] and the angles $\Theta$ (Fig. 2) were found to be constant for a given temperature over the investigated temperature range, that justifies the assumption of a steady-state motion of the entire grain boundary system. It was found that the vertex angle $\Theta$ at the triple junction can deviate distinctly from the equilibrium value, when a low mobility of the triple junction hinders the motion of the grain boundaries. In fact, a transition from triple junction kinetics to grain boundary kinetics was observed (Figs 4 and 5).

\section{THE NATURE OF TRIPLE-JUNCTION DRAG}

As mentioned, the triple junction may have a line energy, just like a dislocation [1, 11]. At least for the case of triple junctions between periodically

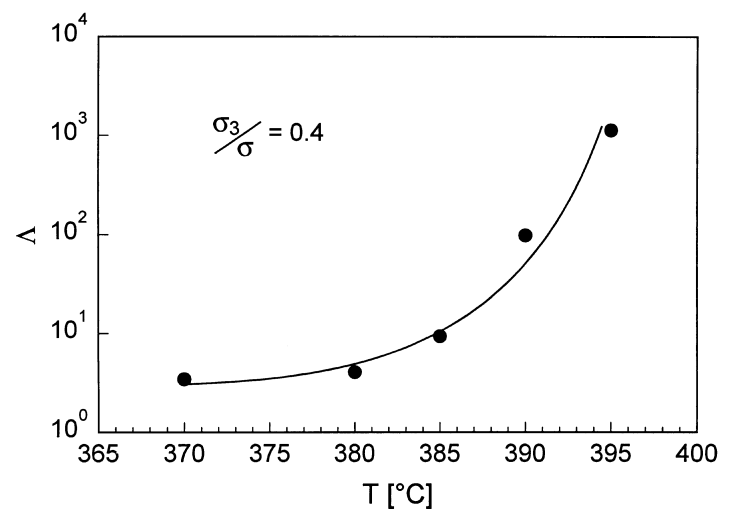

Fig. 4. Temperature dependence of the criterion $\Lambda$ for a symmetrical triple junction [9].

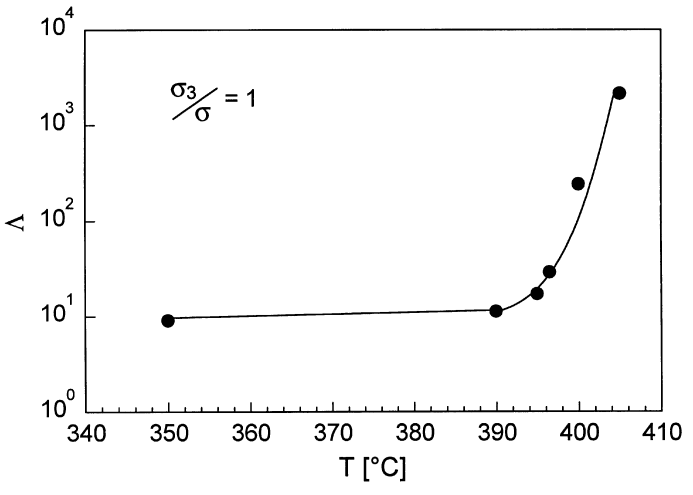

Fig. 5. Temperature dependence of the criterion $\Lambda$ for an ideal triple junction [9].

structured grain boundaries, the junction structure is a periodic junction of its position. If the boundaries are coincidence-related, then the triple-junction structure varies with the same period as that of the largest coincidence-site lattice (CSL) among the three boundaries. This structure variation produces an energy variation with position, and a resulting force (per unit length) that acts upon the triple junction

$$
F=\nabla W
$$

where $W$ is the energy per unit length of the triplejunction line. Since the energy of the triple junction varies periodically with position, so does this selfforce acting upon it: the energy and the force are exactly analogous to the Peierls energy and the Peierls force for a lattice dislocation.

When no external force is applied to the triple junction, we presume that it seeks its local minimum-energy position and the force acting upon it goes to zero. Under these conditions, the forces applied by the grain boundaries must sum to zero and, in the isotropic approximation, the dihedral angles are given by the usual sine-law relationship, which can be expressed as a vector diagram as shown in Fig. 6(a). As the junction starts to respond to tractions applied by the grain boundaries, however, the self-force increases and must resist the motion. Only when the externally applied force exceeds the maximal value of the self-force will the triple junction move over its energy maximum in the direction of motion. The driving force available to move the triple junction is exactly the difference between the applied force and the resistance force (or "drag force"), which is seen to be the maximal value of $F$, designated as $F_{\text {drag. }}$. The forces acting on the triple junction at the point where motion commences are then as shown in Fig. 6(b). We see that the drag force acts to change the dihedral angles, which must reach a critical value before the triple junction will start to move. In fact, we can use the change in dihedral angle between the 


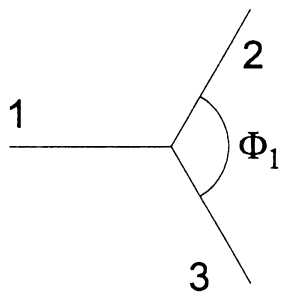

(a)

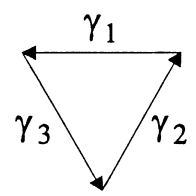

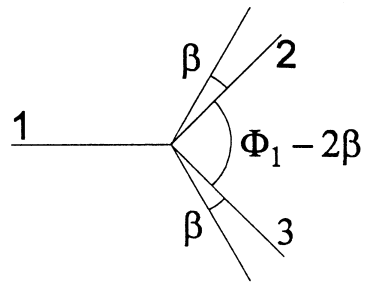

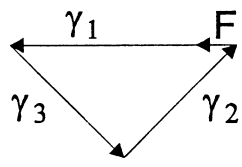

(b)

Fig. 6. The forces acting upon a triple junction: (a) equilibrated, at rest; (b) as the dihedral angle changes. $F$ increases in response to the changing dihedral angle until it reaches its maximal value, $F_{\text {drag. }}$.

static and dynamic structures, to get a direct measure of the drag force magnitude.

This consideration of the physical origin of the drag force indicates the same general behavior as that deduced in Ref. [10]. We conclude that triplejunction motion is akin to Bingham flow: linearly dependent upon driving force, after a threshold force (or dihedral angle) is exceeded, as shown schematically in Fig. 7.

Periodic drag forces result intrinsically from the structures of triple junctions in homogeneous, pure materials. Non-periodic drag forces can be caused by local structural features such as precipitates, surface pits or solute atmospheres. In these cases, additional drag forces may apply, and these act in the same way as the breakaway force that applies to grain boundary migration under the influence of solute drag [12-15]. The triple junction will appear to have two different mobilities, above and below this threshold driving force.

\section{THE EFFECT OF TRIPLE-JUNCTION DRAG ON VON NEUMANN-MULLINS RELATION}

\subsection{Phenomenological approach}

Let us consider how the mentioned results influence the Von Neumann-Mullins relation (6). For this we assume that the influence of the triple junction is rather large, but, nevertheless, the motion of the system can be viewed as grain boundary motion, since the driving force is still due to the grain boundary curvature, i.e. the role of the triple junction is reduced to a change of the angle $\Theta \dagger$. As can be seen from Fig. 2, the model configuration correlates to grains in a polycrystal with less than

\footnotetext{
$\uparrow$ As mentioned above equation (6) describes the steadystate value of the angle $\Theta$. Of course, triple junctions in real polycrystals rarely experience steady-state motion. However, the attainment of a true steady state is not important in this context. Even if the angle $\Theta$ is not in steady state with the moving triple junction, it will be different from the equilibrium $\Theta$ angle $\pi / 3$ as assumed for the Von Neumann-Mullins relation and thus, affect the kinetics with the same tendency as in steady state.
}

six neighbors (adjacent grains), in other words, the topological class of the grain is smaller than six.

For grains with topological class greater than six let us consider the steady-state motion of a grain boundary system shown in Fig. 8 with the same set of assumptions applied to the previous boundary system, namely, uniform grain boundary properties and quasi-two-dimensionality [10]. The steady-state motion of this system is determined by

$$
y(x)=-\frac{x_{0}}{\ln \sin \Theta} \arccos \left(\mathrm{e}^{\left(x / x_{0}\right) \ln \sin \Theta}\right) .
$$

The velocity of the triple-junction motion can be expressed as

$$
V_{\mathrm{Tj}}=m_{\mathrm{Tj}} \sigma(1-2 \cos \Theta) .
$$

The velocity of steady-state motion of the system is

$$
V=-\frac{m_{\mathrm{b}} \sigma}{x_{0}} \ln \sin \Theta .
$$

The length $x_{0}$ replaces the role of the grain size $a$ in the previous case (Fig. 8) or

$$
\begin{aligned}
y_{0} & =y\left(x_{0}\right)=-\frac{x_{0}}{\ln \sin \Theta} \arccos \left(\mathrm{e}^{\ln \sin \Theta}\right) \\
& =-\frac{x_{0}}{\ln \sin \Theta}(\pi / 2-\Theta) .
\end{aligned}
$$

From equations (9) and (10) we obtain $\Lambda$, which

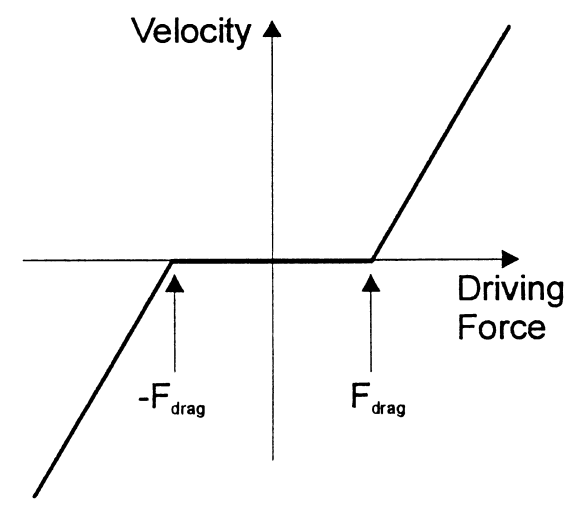

Fig. 7. Schematic illustration of the effect of the drag force on the motion of a triple junction. 


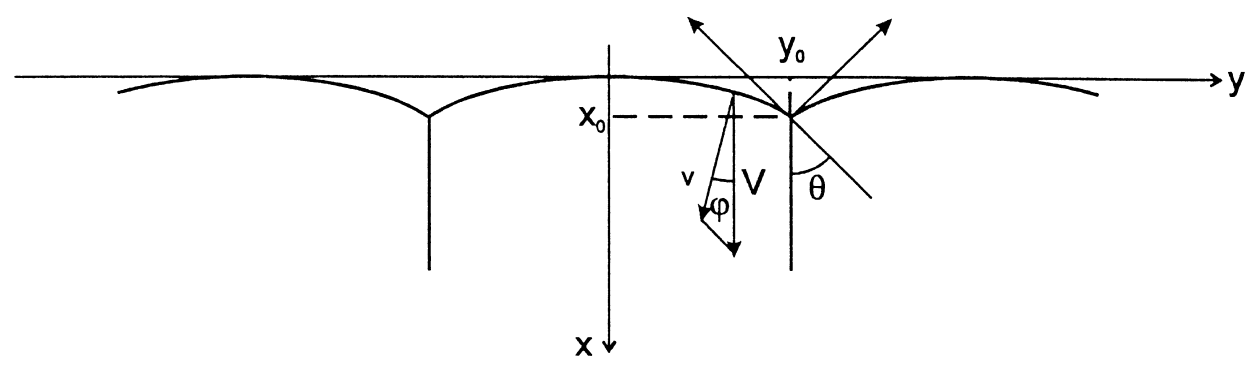

Fig. 8. Configuration of grain boundaries at triple junctions during steady-state motion for $n>6$ [10].

describes the influence of the triple-junction mobility on grain boundary migration

$$
-\frac{\ln \sin \Theta}{1-2 \cos \Theta}=\frac{m_{\mathrm{Tj}} x_{0}}{m_{\mathrm{b}}}=\Lambda \text {. }
$$

Obviously, for $\Lambda \gg 1$, when the boundary mobility determines the kinetics of the system the angle $\Theta$ tends to its equilibrium value $(\pi / 3)$.

Again, the angle $\Theta$ changes when a low mobility of the triple junction starts to drag the motion of the boundary system. However, as can be seen from equation (12) and Fig. 3, in this case the steadystate value of the angle $\Theta$ increases as compared with the equilibrium state. (Otherwise the triple junction would move in the negative direction of the $x$-axis, increasing the free energy of the system.)

For $\Lambda \ll 1$ the angle $\Theta$ [equation (12)] tends to approach $\pi / 2$. The dependency $\Theta=\Theta(\Lambda)$ for both $n<6$ and $n>6$ are shown in Fig. 3 .

The rate of area change for a grain with $n<6$ can be expressed as

$$
\begin{aligned}
\frac{\mathrm{d} S}{\mathrm{~d} t} & =-m_{\mathrm{b}} \sigma \oint \mathrm{d} \varphi=-A_{\mathrm{b}}(2 \pi-n(\pi-2 \Theta)) \\
& =A_{\mathrm{b}}(n-2 \Theta)\left(n-\frac{2 \pi}{\pi-2 \Theta}\right)
\end{aligned}
$$

Since the limited mobility of the triple junction reduces the steady-state value of the angle $\Theta$ as compared with the equilibrium angle, the shrinking rate of grains with $n<6$ decreases, as is obvious for the case when the mobility of the triple junction becomes very low. In other words, for grains with $n<6$ the influence of the triple-junction mobility slows down the process of grain structure evolution, decreasing the vanishing rate of grains with small topological class $(n<6)$.

For grains with topological class greater than six let us refer to the considered steady-state motion of a grain boundary system with a large number of triple junctions (Fig. 8) [10]. The dimensionless parameter $\Lambda$, which describes the influence of the triple-junction mobility on grain boundary migration for such a system is given by equation (12). When a low mobility of the triple junction starts to drag the motion of the boundary system, the angle $\Theta$ changes. However, in this case, the steady-state value of the angle $\Theta$ increases as compared with the equilibrium state. Such an increase of the angle $\Theta$ also decreases the magnitude of $(\pi-2 \Theta)$ in equation (13), in other words, it decreases the "effective" magnitude of the topological class of the growing grain with $n>6$. Consequently, microstructural evolution will slow down due to triplejunction drag for any $n$-sided grain.

The only exception holds for $n=6$, since a hexagonal grain structure becomes unstable when the contact angle $2 \Theta \neq 2 \pi / 3$. Since the actual magnitude of $\Theta$ is determined by the triple junction and grain boundary mobility as well as the grain size and is independent of the number of sides of a grain, there is no unique dividing line between vanishing and growing grains with respect to their topological class anymore, like $n=6$ in the Von Neumann-Mullins approach.

\subsection{Geometrical approach}

Let us rationalize the Von Neumann-Mullins growth law [2,3] simply, as follows: grain growth derives from curvature-driven grain boundary migration, and the grain boundary curvature derives from the preservation of particular dihedral angles at the triple junctions. With uniform, isotropic grain boundary energy the dihedral angles are all $2 \pi / 3$. Thus in two dimensions, grains with more than six sides have convexly curved grain boundaries, grains with less than six sides are concave, and six-sided grains have flat boundaries. This results in the growth of large grains and the shrinkage of small ones that we recognize as conventional grain growth.

Let us now consider the shrinkage of small grains (having less than six sides) in a polycrystalline matrix. In the two-dimensional case, with uniform grain boundary energy and mobility, and with grain boundary migration driven only by curvature, the rate of area loss of a grain is constant, provided that the grain maintains a constant shape. We can therefore study the effects of varying dihedral angles independently of other variables. We present the analysis for grains of two, three, four and five sides (Fig. 9).

When triple-junction drag affects the process, as 


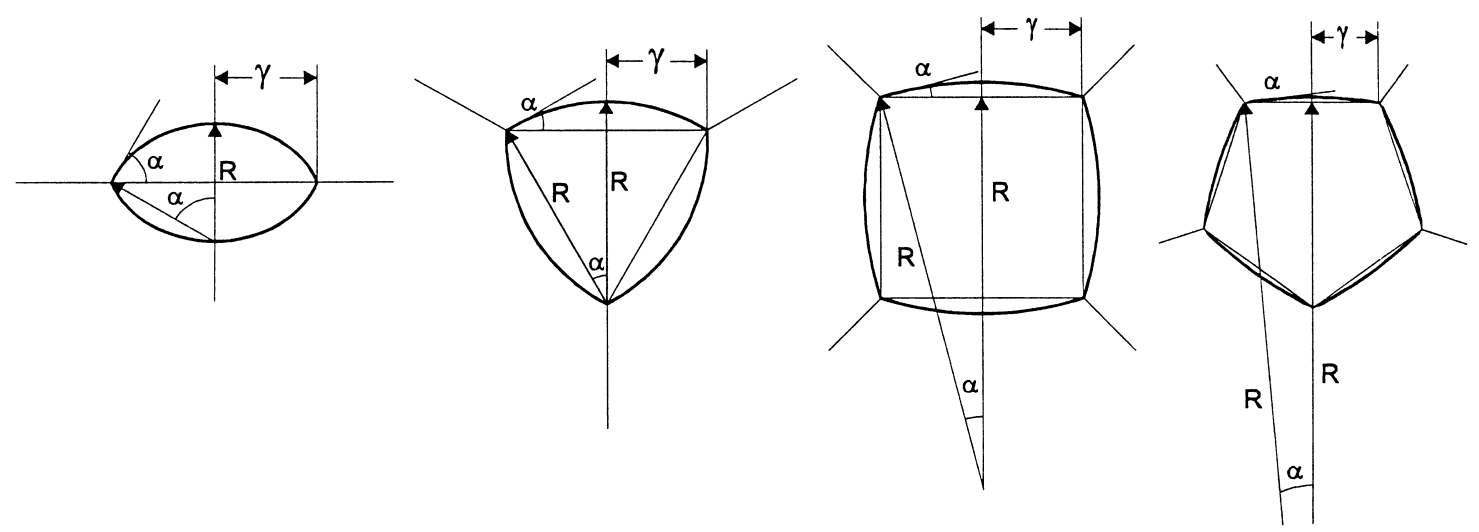

Fig. 9. The geometry of two-, three-, four- and five-sided grains, as used in the analysis presented in the text. The grain boundaries are cylindrical surfaces, with radius $R$.

discussed above, the angles $\alpha_{i}$ are reduced by an amount related to the drag force. We denote this angle as $\beta$, and it is half of the reduction in the dihedral angle. Thus, under the influence of triplejunction drag, we have $\alpha_{2}=60^{\circ}-\beta ; \alpha_{3}=30^{\circ}-\beta$; $\alpha_{4}=15^{\circ}-\beta ; \alpha_{5}=6^{\circ}-\beta$. Larger drag forces produce larger values of $\beta$. We can now compute the effect of increasing triple-junction drag upon the rate of shrinkage of these various types of small grains. Without performing any computations at all, however, it is quite easy to see what the effect of increasing the drag term will be. In the case of a five-sided grain, $\alpha_{5}=6^{\circ}-\beta$, so we can readily see that a $\beta$-value of $6^{\circ}$ results in a shrinkage rate of zero. The reason for this is simple: once this value is reached, the grain has flat sides that no longer migrate inward. Effectively, then, this five-sided grain with draggy triple junctions acts like a sixsided grain in the classical Von Neumann-Mullins analysis.

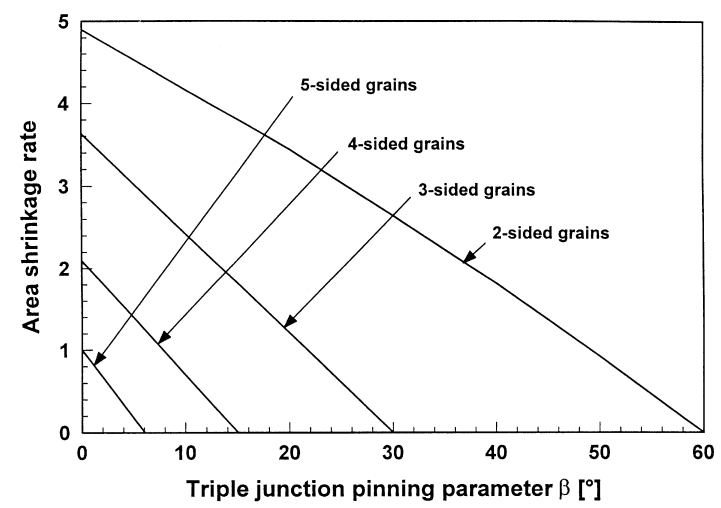

Fig. 10. The area rate of shrinkage (in units of $A_{\mathrm{b}}$ ) for two-, three-, four- and five-sided grains, as a function of the critical inclination angle shift, $\beta . \beta$ increases with the triple-junction drag force, and it can be seen that increasing the drag force retards the shrinkage of these small grains. The growth of large grains is similarly affected, and the process of grain growth is generally retarded by triple-junction drag.
The relative rates of shrinkage for the different grains are shown in Fig. 10, as a function of $\beta$. It is clear that increasing drag force reduces the shrinkage rates of all of these grains. As the drag force increases, first five-sided grains become stable, then four-sided ones, etc. Two-sided grains can never be stabilized, no matter how large the triple-junction drag force, since that requires a $\beta$-value of $60^{\circ}$, which makes the dihedral angle go to zero, removing the grain before triple-junction motion occurs.

\section{CONCLUSIONS}

The effect of triple-junction mobility on the rate of change of the grain area during grain growth was investigated. It was found that a finite junction mobility exerts a drag on the adjoining grain boundaries. This is reflected by a deviation of the grain vertex angles at triple junctions from their equilibrium value $2 \pi / 3$ and correspondingly, by a modification of the Von Neumann-Mullins relation. It was shown that for the situation when the triple junction influence on grain boundary motion is large enough, but nevertheless the grain boundary motion is controlled by grain boundary kinetics, the triple junction influence results in a reduced rate of microstructure evolution during grain growth. The results of the geometrical approach correlate well with conclusions of phenomenological considerations. One of the main consequences of the phenomenological consideration relates to the stability of a hexagonal grain structure under the conditions when the low mobility of the triple junctions drags the grain boundary motion. Since the actual magnitude of the angle at a tip of a triple junction is determined by the triple junction and grain boundary mobility as well as the grain size and does not depend on the number of sides of a grain, there is no unique border line between vanishing and growing grains with respect to their topological class anymore, like $n=6$ in the Von Neumann-Mullins approach. 
Acknowledgements-Financial support from the Deutsche Forschungsgemeinschaft (Grant No. 436 RUS113/539/0) and Russian Fundamental Research Foundation (Grant No. 990204017) are gratefully acknowledged. One of the authors (Alex King) is grateful for the support of the US National Science Foundation, grant No. DMR-9530314, in the performance of this work.

\section{REFERENCES}

1. Gibbs, J. W., Collected Works, in Two Volumes. Longmans, Green, New York, 1928.

2. Weygand, D., Brechet, Y. and Lepinoux, J., Acta mater., 1998, 46, 6559.

3. Von Neumann, J., in Metal Interfaces. American Society for Testing Materials, Cleveland, OH, 1952, p. 108

4. Mullins, W. W., J. appl. Phys., 1956, 27, 900.

5. Lücke, K., Heckelmann, I. and Abbruzzese, G., Acta metall. mater., 1992, 40, 533.

6. Anderson, M. P., Srolovitz, D. J., Grest, G. S. and Sahni, P., Acta metall., 1984, 32, 783.

7. Fradkov, V. E., Shvindlerman, L. S. and Udler, D. G., Scripta metall., 1985, 19, 1285.

8. Fradkov, V. E. and Shvindlerman, L. S., in Structure and Properties of Interfaces in Metals. Nauka, Moscow, 1988, p. 213.

9. Galina, A. V., Fradkov, V. E. and Shvindlerman, L. S., Physics Metals Metallogr., 1987, 63, 165.

10. Czubayko, U., Sursaeva, V. G., Gottstein, G. and Shvindlerman, L. S., Acta mater., 1998, 46, 5863.

11. Gottstein, G. and Shvindlerman, L. S., Scripta mater., 1998, 38, 1541

12. King, A. H., in Boundaries \& Interfaces in Materials: The David A. Smith Symposium, eds. R. C. Pond, W A. T. Clark, A. H. King and D. B. Williams. TMS, Warrendale, PA, 1998, p. 151.

13. Lücke, K. and Detert, K., Acta metall., 1957, 5, 628.

14. Cahn, J. W., Acta metall., 1962, 10, 789.

15. Lücke, K. and Stüwe, H. P., Acta metall., 1971, 19 , 1087.

\section{APPENDIX}

A one-sided grain is circular, with radius $R$, and with no triple junctions. The curvature is $1 / R$ and the area is $R^{2}$. Writing the velocity of the grain boundary as

$$
V=A_{\mathrm{b}} \frac{1}{R}
$$

we deduce that

$$
\frac{\mathrm{d} R}{\mathrm{~d} t}=A_{\mathrm{b}} \frac{1}{R} .
$$

Since

$$
\begin{gathered}
\frac{\mathrm{d} S}{\mathrm{~d} R}=2 \pi R \\
\frac{\mathrm{d} S}{\mathrm{~d} t}=-2 \pi A_{\mathrm{b}} .
\end{gathered}
$$

Thus the rate at which cross-sectional area is lost from the shrinking grain to the surrounding grain is a constant, and simply related to the grain boundary mobility.

A two-sided grain is illustrated in Fig. 3. The area of the grain is

$$
S_{2}=2 R^{2}\left(\alpha_{2}-\sin \alpha_{2} \cos \alpha_{2}\right) .
$$

If the grain shrinks self-similarly

$$
\frac{\mathrm{d} R}{\mathrm{~d} t}=A_{\mathrm{b}} \frac{1}{R\left(1-\cos \alpha_{2}\right)} .
$$

Then

$$
\frac{\mathrm{d} S_{2}}{\mathrm{~d} t}=-4 A_{\mathrm{b}} \frac{\left(\alpha_{2}-\sin \alpha_{2} \cos \alpha_{2}\right)}{\left(1-\cos \alpha_{2}\right)} .
$$

For a three-sided grain, we obtain by a similar process

$$
\frac{\mathrm{d} S_{3}}{\mathrm{~d} t}=-6 A_{\mathrm{b}} \frac{\left(\alpha_{3}-\sin \alpha_{3} \cos \alpha_{3}+\sin ^{2} \alpha_{3} / \sqrt{3}\right)}{\left(1+\frac{\sin \alpha_{3}}{2}-\cos \alpha_{3}\right)} .
$$

For a four-sided grain

$$
\frac{\mathrm{d} S_{4}}{\mathrm{~d} t}=-8 A_{\mathrm{b}} \frac{\left(\alpha_{4}-\sin \alpha_{4} \cos \alpha_{4}+\sin ^{2} \alpha_{4}\right)}{\left(1+\sin \alpha_{4}-\cos \alpha_{4}\right)} .
$$

The result for a five-sided grain is

$$
\frac{\mathrm{d} S_{5}}{\mathrm{~d} t}=-10 A_{\mathrm{b}} \frac{\left(\alpha_{5}-\sin \alpha_{5} \cos \alpha_{5}+1.3764 \sin ^{2} \alpha_{5}\right)}{\left(1+1.3764 \sin \alpha_{5}-\cos \alpha_{5}\right)} .
$$

In each of these cases, the angle $\alpha_{i}$ is the internal angle of the circular arc that defines the grain boundary position. If the dihedral angles are all $2 \pi /$ 3 , then the respective values of $\alpha_{i}$ are $\alpha_{2}=60^{\circ}$; $\alpha_{3}=30^{\circ} ; \alpha_{4}=15^{\circ} ; \alpha_{5}=6^{\circ}$. 